\title{
Ellipsometric measurements by use of photon pairs generated by spontaneous parametric downconversion
}

\author{
Ayman F. Abouraddy, Kimani C. Toussaint, Jr., Alexander V. Sergienko, Bahaa E. A. Saleh, and Malvin C. Teich \\ Quantum Imaging Laboratory, Departments of Electrical \& Computer Engineering and Physics, Boston University, \\ Boston, Massachusetts 02215-2421
}

Received May 21, 2001

\begin{abstract}
We present a novel interferometric technique for performing ellipsometric measurements. This technique relies on the use of a nonclassical optical source, namely, polarization-entangled twin photons generated by spontaneous parametric downconversion from a nonlinear crystal, in conjunction with a coincidence-detection scheme. Ellipsometric measurements acquired with this scheme are absolute; i.e., they do not require source and detector calibration. (C) 2001 Optical Society of America

OCIS codes: $120.2130,270.0270,190.0190,120.3940,000.160,350.4600$.
\end{abstract}

Ellipsometry ${ }^{1-6}$ is a well-established metrological technique that is used, particularly in the semiconductor industry, to determine the thickness and optical constants of thin films. Because of the high accuracy required in measuring these parameters, an ideal ellipsometric measurement would require absolute calibration of both the source and the detector. As this is not attainable in practical settings, ellipsometry makes use of a myriad of experimental techniques developed to circumvent the imperfections of the devices involved. The most common of these techniques are null and interferometric ellipsometry.

In this Letter we demonstrate how to satisfy the aforementioned requirements by using a nonclassical source of light: a two-photon polarization-entangled state generated by type II spontaneous parametric downconversion (SPDC). ${ }^{7,8}$ This source has been used in the emerging field of quantum metrology. ${ }^{9} \mathrm{We}$ show that, by utilizing the quantum correlations exhibited by the photon pairs in such a state, one may obtain absolute ellipsometric data from a sample. This is done in a simple setting with a minimal number of optical components.

In the traditional (classical) null ellipsometer the sample is illuminated with a beam of light that can be prepared in any state of polarization. The reflected light, which is generally elliptically polarized, is then analyzed. The polarization of the incident beam is adjusted to compensate for the change in the relative amplitude and phase between the two eigenpolarizations introduced by the sample such that the resultant beam is linearly polarized. If it is passed through an orthogonal linear polarizer, this linearly polarized beam will yield a null (zero) intensity at an optical detector. The null ellipsometer does not require a calibrated detector because it does not measure intensity but instead records a null. The principal drawback of null measurement techniques is the need for a reference against which to calibrate the null, for example, to find its initial location (the rotational axis of reference at which an initial null is obtained) and compare it with the subsequent location when the sample is inserted into the device. The accuracy and reliability of all measurements crucially depend on the accuracy and reliability of our knowledge of the reference sample.

A traditional (classical) alternative is to employ an interferometric configuration in which the light from the source follows more than one path to the detector. The sample is placed in one of those paths. The overall throughput of the system (both source and detector parameters) is estimated by removal of the sample. This technique does not require a reference sample but relies on knowledge of the detector's efficiency. Interferometric configurations employ optical components (such as beam splitters) that require accurate characterization and in general increase instrumental errors.

The proposed entangled-photon quantum ellipsometer is illustrated in Fig. 1. An intense laser (pump) beam illuminates a birefringement nonlinear optical crystal (NLC). Quantum mechanics predicts that some of the pump photons will disintegrate into pairs, known traditionally as signal and idler, which conserve energy (frequency matching) and momentum (phase matching), ${ }^{8,10}$

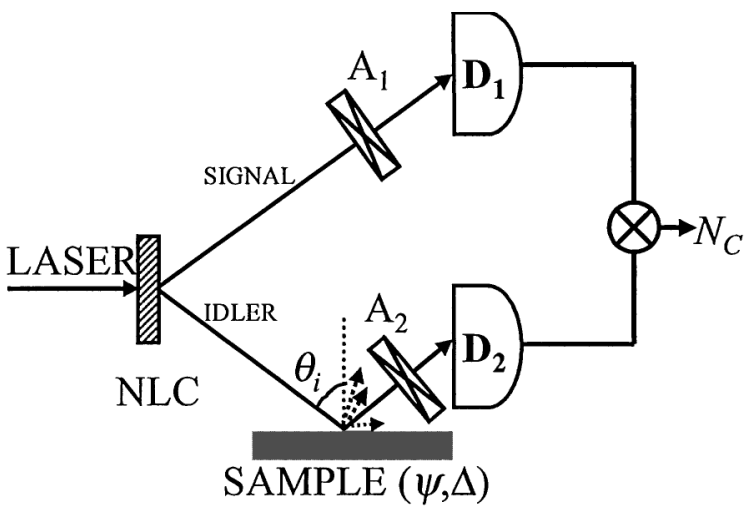

Fig. 1. Entangled-photon quantum ellipsometer. Abbreviations are defined in text. The sample is characterized by the ellipsometric parameters $\psi$ and $\Delta$. 
For our purposes, we choose the SPDC to be in a configuration known as type II noncollinear. Type II refers to the fact that the signal and idler photons have orthogonal polarizations (ordinary and extraordinary) to satisfy the phase-matching conditions; the term "noncollinear" indicates that the signal and the idler photons are emitted in two different directions. Because of the birefringence of the NLC that is used to generate SPDC, the signal and idler photons emerge from the NLC with a relative time delay that one compensates for by placing an appropriate birefringent material of suitable thickness in the signal and (or) idler path. ${ }^{7}$ Specifically, in type II SPDC from a negative uniaxial NLC (such as beta-barium borate), the signal photon (extraordinary polarized) emerges from the NLC before the idler photon (ordinary polarized). One compensates for this by placing a quartz plate, for example, of suitable thickness in one of the arms of the setup. The exit surface of the NLC typically has an antireflection coating to reduce reflection losses of the two polarizations.

The signal and idler photons are emitted in a polarization-entangled state described by ${ }^{7,8}$

$$
|\Psi\rangle=(1 / \sqrt{2})(|H V\rangle+|V H\rangle),
$$

where $H$ and $V$ represent horizontal and vertical polarizations, respectively. It is understood that the first polarization in any ket is that of the signal photon and the second is that of the idler. Although the twophoton entangled state is a pure quantum state, the signal and the idler photons considered separately are each unpolarized. ${ }^{11,12}$ As shown in Fig. 1, the signal beam encounters a linear polarization analyzer $\left(A_{1}\right)$, followed by a single-photon photodetector $\left(D_{1}\right)$. The idler beam reflects off the sample of interest before it encounters a linear polarizer $\left(\mathrm{A}_{2}\right)$ followed by single-photon photodetector $\left(\mathrm{D}_{2}\right)$. The sample is characterized by the parameters $\psi$ and $\Delta: \psi$ is the ratio of the magnitudes of the sample reflection coefficients, $R_{H}$ and $R_{V}$ for the $p$ - and $s$-polarized waves, respectively; $\Delta$ is the phase shift between them. The detectors are part of a circuit that records the coincidence rate of photon pairs.

The nonclassical source and the optical arrangement shown in Fig. 1 exhibit two features that circumvent the two problems noted above, i.e., calibration of the source and of the detector. The first characteristic of our proposed quantum ellipsometer is that the source is a twin-photon source; i.e., we are guaranteed on detection of one photon in one of the arms of the setup that its twin is in the other. The detection of one photon may be used to gate the arrival of its twin in the other arm, and thus we are effectively provided with a calibrated optical source because the efficiency of the gating detector is immaterial. The second characteristic is the polarization entanglement of the source. Polarization entanglement acts as an interferometer in our approach, thereby alleviating the need for calibrating the second detector in our coincidence scheme.
The coincidence rate, $N_{c}$, recorded by $\mathrm{D}_{1}$ and $\mathrm{D}_{2}$ is proportional to the fourth-order coherence function ${ }^{13,14}$ and is given by

$$
\begin{aligned}
N_{c}= & C\left|\beta e^{j \Delta} \cos \theta_{1} \sin \theta_{2}+\sin \theta_{1} \cos \theta_{2}\right|^{2} \\
\propto & \beta^{2} \cos ^{2} \theta_{1} \sin ^{2} \theta_{2}+\sin ^{2} \theta_{1} \cos ^{2} \theta_{2} \\
& +2 \beta \cos \Delta \cos \theta_{1} \sin \theta_{1} \cos \theta_{2} \sin \theta_{2} .
\end{aligned}
$$

Here $C$ is a constant that includes the quantum efficiency of the detectors and the various parameters of the experimental arrangement, $\beta=\sqrt{\tan \psi}$, and $\theta_{j}(j=1,2)$ is the angle of the analyzer with respect to $H$. If the sample is replaced by a perfect mirror, the coincidence rate is a sinusoidal pattern of $100 \%$ visibility. In practice, by judicious control of the apertures placed in the downconverted beams, visibilities close to $100 \%$ can be obtained.

One may use relation (2) to extract ellipsometric data by fixing one of the analyzers and rotating the other. It is advantageous to fix analyzer $\mathrm{A}_{2}$ in the sample arm and rotate $A_{1}$. One may choose $\theta_{2}=45^{\circ}$, for example, whereupon

$$
N_{c}=(C / 2)\left|\beta e^{j \Delta} \cos \theta_{1}+\sin \theta_{1}\right|^{2} .
$$

Three angles of $\mathrm{A}_{1}$ are sufficient for estimating the three parameters $C, \psi$, and $\Delta$ (an obvious choice would be $\theta_{1}=0^{\circ}, 45^{\circ}, 90^{\circ}$ ). It is sometimes advantageous to choose a different value for $\theta_{2}$ to equalize the two terms in the first line of relation (2), particularly if $\beta \gg 1$ or $\beta \ll 1$.

An important feature of this interferometer is that it is not sensitive to an overall mismatch in the length of the two arms of the setup. In this case one can show that the coincidence rate is identical to that given in relation (2), regardless of the mismatch.

Another advantage of this setup over its idealized null ellipsometric counterpart is that the two arms of the ellipsometer are separate and the light beams traverse them independently in different directions. This configuration allows various instrumentation errors of the classical setup to be circumvented. The advantage of placing all optics in the idler channel arises from the fact that optical alignment is easier to achieve in a transmission, rather than in a reflection, configuration. The system is less prone to beam-deviation error than is its counterpart. ${ }^{15}$ In our case no optical components are placed between the source (the NLC) and the sample; any desired polarization manipulation may be performed in the other arm of the entangled-photon ellipsometer.

A significant drawback of classical ellipsometry is the difficulty of fully controlling the polarization of the incoming light. A linear polarizer is usually employed 


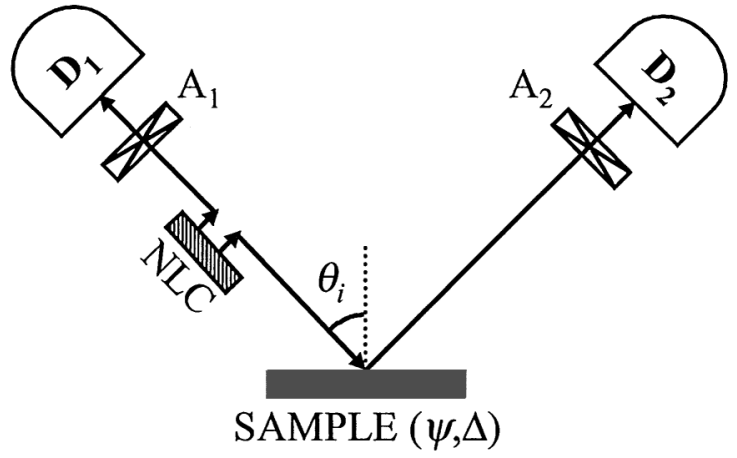

Fig. 2. Unfolded version of the entangled-photon quantum ellipsometer displayed in Fig. 1.

at the input of the ellipsometer, but the finite extinction coefficient of this polarizer causes errors in the estimated parameters. ${ }^{2}$ In the quantum ellipsometer the polarization of the incoming light is dictated by the phase-matching conditions of the nonlinear interaction in the NLC. The polarizations defined by the orientation of the optical axis of the NLC act as the input polarization in classical ellipsometry. The NLC is aligned for type II SPDC, so only one polarization component of the pump generates SPDC, whereas the orthogonal (undesired) component of the pump does not (because it does not satisfy the phase-matching conditions). The advantage is therefore that the downconversion process ensures the stability of polarization along a particular direction.

There are also important practical advantages in employing quantum ellipsometry. All optics (in this setting only analyzer A1) is placed in a path that does not include the sample. Because A2 is the only optic in the sample arm, and, as it follows the sample, one can change the angle of incidence to the sample easily and repeatedly.

An illuminating way to represent the action of the entangled-photon quantum ellipsometer is provided by the redrawing of Fig. 1 in the unfolded configuration shown in Fig. 2. Using the advanced wave interpretation, which was suggested by Klyshko in the context of two-photon imaging, ${ }^{16}$ one may obtain the coincidence rate for photons at $\mathrm{D}_{1}$ and $\mathrm{D}_{2}$ by tracing light waves originating from $\mathrm{D}_{1}$ to the NLC and then onto $\mathrm{D}_{2}$ on reflection from the sample. With this interpretation, the configuration in Fig. 2 becomes geometrically similar to that of the classical ellipsometer. Although none of the optical components usually as- sociated with interferometers (beam splitters and wave plates) is present in this scheme, interferometry is still performed through the entanglement of the source and coincidence measurements.

In summary, we have shown that, by employing entangled-photon pairs that are generated by type II SPDC in a noncollinear configuration, one can obtain absolute ellipsometric data from a reflective sample. The underlying physics that permits such ellipsometric measurements resides in the fact that fourth-order (coincidence) quantum interference of the photon pairs, in conjunction with polarization entanglement, emulates an idealized classical ellipsometric setup that utilizes a source and a detector that are both calibrated absolutely.

This study was supported by the National Science Foundation and by the Center for Subsurface Sensing and Imaging Systems, a National Science Foundation engineering research center. A. V. Sergienko's e-mail address is alexserg@bu.edu.

\section{References}

1. P. Drude, Ann. Phys. Chem. 39, 481 (1890).

2. R. M.A. Azzam and N. M. Bashara, Ellipsometry and Polarized Light (North-Holland, Amsterdam, 1977).

3. H. G. Tompkins and W. A. McGahan, Spectroscopic Ellipsometry and Reflectometry (Wiley, New York, 1999).

4. A. Rothen, Rev. Sci. Instrum. 16, 26 (1945).

5. A. B. Winterbottom, Trans. Faraday Soc. 42, 487 (1946).

6. M. Mansuripur, Opt. Photon. News 11(4), 52 (2000).

7. P. G. Kwiat, K. Mattle, H. Weinfurter, A. Zeilinger, A. V. Sergienko, and Y. Shih, Phys. Rev. Lett. 75, 4337 (1995).

8. D. N. Klyshko, Photons and Nonlinear Optics (Gordon \& Breach, New York, 1988).

9. D. Branning, A. L. Migdall, and A. V. Sergienko, Phys. Rev. A 62, 063808 (2000).

10. B. E. A. Saleh and M. C. Teich, Fundamentals of Photonics (Wiley, New York, 1991).

11. U. Fano, Rev. Mod. Phys. 29, 74 (1957).

12. A. F. Abouraddy, B. E. A. Saleh, A. V. Sergienko, and M. C. Teich, Phys. Rev. A 64, 050101 (2001).

13. R. J. Glauber, Phys. Rev. 130, 2529 (1963).

14. B. E. A. Saleh, A. F. Abouraddy, A. V. Sergienko, and M. C. Teich, Phys. Rev. A 62, 043816 (2000).

15. J. R. Zeidler, R. B. Kohles, and N. M. Bashara, Appl. Opt. 13, 1938 (1974).

16. D. N. Klyshko, Sov. Phys. JETP 67, 1131 (1988) [Zh. Eksp. Teor. Fiz. 94, 82 (1988)]. 